\title{
Labyrinthe
}

17 | 2004 (1)

Jacques Rancière, l'indiscipliné

\section{Police, politique, monde : quelques questions}

\section{Renaud Pasquier}

\section{OpenEdition}

Journals

Édition électronique

URL : http://journals.openedition.org/labyrinthe/168

DOI : $10.4000 /$ labyrinthe. 168

ISSN : 1950-6031

Éditeur

Hermann

Édition imprimée

Date de publication : 15 avril 2004

\section{Référence électronique}

Renaud Pasquier, «Police, politique, monde : quelques questions », Labyrinthe [En ligne], 17 | 2004 (1),

mis en ligne le 15 juillet 2008, consulté le 02 mai 2019. URL : http://journals.openedition.org/

labyrinthe/168; DOI : 10.4000/labyrinthe.168 


\title{
POLICE, POLITIQUE, MONDE : QUELQUES QUESTIONS
}

Renaud PASQUIER

\begin{abstract}
Thèse 6: Il y a deux manières de compter les parties de la communauté. La première ne compte que des parties réelles, des groupes effectifs définis par les différences dans la naissance, les fonctions, les places et les intérêts qui constituent le corps social, à l'exclusion de tout supplément. La seconde compte « en plus » une part des sans-part. On appellera la première police, la seconde politique (« Dix thèses sur le politique », $A B, 239$ ).

Il y a une police mondiale et elle peut parfois procurer quelques biens. Mais il n'y a pas de politique mondiale (Més., 188).
\end{abstract}

La distinction entre police et politique, point névralgique du travail de Rancière, en est sans doute aussi l'élément le plus discuté. Mais un même défaut affecte souvent l'argumentation de ses détracteurs: confondant police et pouvoir ${ }^{1}$ (là où Rancière s'attache justement à marquer la différence) ou encore les « sans-part» (dont le compte surnuméraire vient bouleverser le « partage du sensible » consensuel) avec les pauvres ${ }^{2}$, ils ignorent la spécificité des définitions de Rancière, et limitent du même coup la portée de leurs critiques.

Qui pourtant n'ont rien d'illégitime, tant l'articulation est problématique. La combinaison d'une irréductibilité (la politique n'est et ne sera jamais police) et d'un lien nécessaire (pas de politique hors de la police) risque deux radicalisations: la première, qui considère avant tout l'irréductibilité, conduira au soupçon de toute institutionnalisation, voire à une mystique de l'événement, une sorte d'exaltation de la « révolution permanente »; à l'inverse, la seconde insistera sur le caractère éphémère des subjectivations politiques, et se muera en fatalisme devant leur résorption dans l'ordre inégalitaire - « ... et tu retourneras à la police ». Ces deux positions, également repoussées par Rancière,

1. Voir par exemple Slavoj Zizek, « Le malaise dans la subjectivation politique », Actuel Marx $\mathrm{n}^{\circ} 28$, «Y a-t-il une pensée unique en philosophie politique?», $2^{\mathrm{e}}$ semestre 2000, p. 137-152.

2. Voir Yves Michaud, «Les pauvres et leur philosophe », Critique n 601-602, 1997, p. 445. 
pointent cependant la question principale, celle des effets de la politique. À son propos, plusieurs problèmes :

- Sur quoi repose la «mécanique policière », entropie non irréversible à combattre sans cesse ${ }^{3}$ ? Une improbable «nature humaine»? Des « infrastructures »? Quoi qu'il en soit, il y a là une loi transhistorique dont il est difficile de rendre compte.

- Rancière voit dans certaines revendications, de l'ordre du droit, des «luttes policières », distinctes des subjectivations politiques proprement dites, faute de manifester une " démonstration de capacité ». Comment appréhender un tel statut, à quelle aune juger ces interventions?

- Le problème ici en jeu est celui de la «bonne police ». On voudrait la concevoir comme celle qui, affectée par les effractions politiques répétées, laisserait justement ouverte la possibilité de subjectivations. Cela contreviendrait pourtant à l'hétérogénéité police/politique, et à l'imprévisibilité du surgissement politique; se dessinerait en outre à l'horizon une "meilleure des polices » dont Rancière ne veut pas entendre parler. Le problème de l'évaluation des pratiques policières, et singulièrement, gouvernementales, reste intact.

Ces interrogations, Rancière les rencontre néanmoins quand il considère l'époque contemporaine, dont il retient deux caractéristiques : l'exhibition d'une «démocratie consensuelle», soit, dans les figures équivalentes de la fin et du retour de la politique, une pure négation de celle-ci ; l'aspiration confuse à une extension internationale de la « démocratie » ainsi entendue, soit l'un des visages de la sempiternelle mondialisation.

Deux constats qu'il s'agit d'éclairer. Notons-le d'emblée, l'attention aux formes passées de subjectivation fait place à l'analyse inlassable de la configuration consensuelle. Témoin le foisonnement terminologique pour la qualifier: postdémocratie (Més.), ochlocratie, épistémocratie $(A B)$, oligarchie ${ }^{4}$, ploutocratie ${ }^{5}$. Où se lisent l'ampleur et la difficulté de la tâche. De fait, le diagnostic établi est sombre, et notre temps «nihiliste» (Més., 167-188). On se demande alors, la politique étant constitutivement difficile, ce qui la rend encore plus rare aujourd'hui : « Son éclipse actuelle est bien réelle » (Més., 188).

3. Lire «La déviation démocratique », dans Les Transitions démocratiques, Laënnec Heurbon (dir.), 1996.

4. «La déviation démocratique », art. cit.

5. « La guerre comme forme suprême du consensus ploutocratique avancé », Lignes n 12, 2003. 
C'est d'abord le règne du consensus, entendu comme réduction de la politique à la police, perception de la communauté comme stricte somme de ses parties - ici prendrait place l'idée de démarches strictement «policières » qui, faute de favoriser la construction de scènes, ne lui fassent du moins pas obstacle.

C'est ensuite la complexité de ce consensus, qui joue sur deux niveaux:

L'agir politique se trouve en effet pris en tenaille entre les polices étatiques de la gestion et la police mondiale de l'humanitaire (Més., 184).

L'espace mondial n'apparaît qu'à l'horizon du travail de Rancière; il s'impose à lui depuis environ dix ans. Sa réflexion se déploie sur trois plans. D'abord comme critique de la catégorie d' « humanitaire » attachée aux représentations de la «communauté internationale », manifestation ultime de la philosophie politique - de son entreprise de suppression de la politique - sous la forme de l'éthique (Més., 171172). En un second plan, en phase avec l'actualité la plus récente, il examine l'évolution brutale de ce consensus mondial, à savoir, pour reprendre le titre d'un texte de novembre 2003, « La guerre comme forme suprême du consensus ploutocratique avancé ${ }^{6} »$. On y trouve une très incisive et très convaincante analyse de la notion d'insécurité, qui articule les deux niveaux de la police, comme sentiment qu'il importe de nourrir (sous couvert de lutter contre) afin de resserrer les communautés de la peur (et de la haine de l'autre) où la puissance de division qu'est le dèmos disparaît derrière le tout identitaire de l'ethnos. Les luttes contre « les États-voyous » ou les « sauvageons » ne sont autres que des visages du consensus triomphant, « utopie du gouvernement de la planète par l'autorégulation du capital ${ }^{7}$ ", où se confondent pouvoirs étatique, militaire, médiatique et financier.

Le troisième plan de réflexion concerne dès lors les moyens de lutter contre cette utopie mondialiste..., s'il y en a (voir la citation liminaire). L'affirmation d'absence d'une politique mondiale prend la forme d'une critique récurrente des thèses d'Empire, de Negri et $\mathrm{Hardt}^{8}$, qui tentent de prendre en compte la dimension mondiale du consensus - pour conserver le terme de Rancière. Ce dernier y voit un «marxisme vita-
6. Ibid.
7. Ibid., p. 37.
8. Paris, Exils, 2000. 
liste », qui abandonne le peuple pour les multitudes, en une ontologie des sujets politiques qui s'inscrit finalement dans la métapolitique philosophique attaquée par La Mésentente ${ }^{9}$. Ajoutons à cela la sympathie (très) sceptique témoignée par Rancière aux altermondialistes ou au mouvement des sans-papiers ${ }^{10}$, et l'on se figurera l'impasse présente de la politique: dans une configuration mondiale du consensus, elle serait restreinte au cadre national (étatique), donc condamnée. Comme si le caractère local de la politique (Més., 188) glissait d'une désignation de la singularité des cas à une acception géographique. Le couple police/politique semblait pourtant permettre de penser la politique hors de l'État et son cadre national. Le corrélat entre les deux notions paraît puissant: dès lors qu'il y a police mondiale, pourquoi pas de politique?

Deux hypothèses, alors. La première prendrait acte de la nécessité d'un État. Deux options se présentent: soit défendre l'État national contre la «mondialisation », et tomber dans les paradoxes qui entravent la contestation altermondialiste; soit envisager un État mondial, rêve d'une «meilleure des polices » indistinct de l'utopie mondialiste. L'analyse de Rancière en sortirait donc défaite. Dans la seconde hypothèse, on considère que, quoique élaborée dans un cadre nationalétatique, la distinction police/politique est opératoire à d'autres échelles: c'est en somme être plus « ranciérien » que Rancière, et tenir la possibilité d'une «scène mondiale » de la politique; soit la possibilité de subjectivations politiques contre la double liquidation consensuelle du peuple comme agent démocratique: par réduction à l'ethnie (versant national-étatique), par dissolution dans la foule consommatrice (versant mondialiste-capitaliste) ${ }^{11}$. Contre son propre et relatif pessimisme, Rancière ouvre la voie à cette aspiration, en se faisant, sinon prescriptif, du moins plus injonctif qu'à l'ordinaire :

Un mouvement démocratique conséquent doit [...] prendre la pleine mesure de ce qui sépare ses formes des formes de l'État et sa liberté de la liberté des marchandises ${ }^{12}$.

9. Pour un développement de ce débat, on lira deux entretiens : «Peuple ou multitudes ? », Multitudes $\mathrm{n}^{\circ} 9,2002$ et Dissonance, texte en ligne à http://www.messmedia.net/dissonance/issues/ issue01/issue01_13.htm

10. Voir «La communauté comme dissentiment », Rue Descartes n 42, 2003, p. 97-99 et «Dissenting Words », Diacritics volume 30, n², 2000, p. 123-126.

11. Voir «La déviation démocratique », art. cit.

12. « La guerre comme forme suprême du consensus ploutocratique », art. cit., p. 39. 